\title{
THE PERSONALIZATION AND PREDICTION INNOVATION OF MOBILE RECOMMENDER SYSTEMS
}

\author{
Theophilus D. Owusu, Keiser University, towusu@keiseruniversity.edu \\ Christopher Hoffman, Keiser University, choffman@keiseruniversity.edu
}

\begin{abstract}
With the advent of the Internet, the ability to retrieve and use information has become ubiquitous, but the amount of information available has also grown exponentially. This information growth has caused information overload for users trying to sift through the information to find the best options that will meet their needs, thus recommendation systems were developed. The current recommendation systems are rudimentary in function and are basically able to provide suggestions for similar products, local restaurants and hotels, or point-of-interest locations. The future of the recommendation systems, including mobility, will be able to take user behavior and contextual information to make better and more precise suggestions. The new system will be able to protect the user's privacy and personal information along with seamlessly communicating with each other through standardization. This paper will provide current literature research along with the problems and probable solutions to the mobile recommender systems industry.
\end{abstract}

Keywords: Mobile Recommender Systems, Mobile Information Systems, Mobile Searches, Mobile GPS, Mobile GIS, Mobile Search Engines, Mobile Location Searches.

\section{INTRODUCTION}

Recommender systems have been around for many years and have been responsible for increasing profits for companies and institutions, giving pertinent information to users and enterprises. We easily see this within supply chain management systems, enterprise resource planning (ERP) systems, accounting and finance systems and even in hospital information technology (HIT) systems. However, "system" agnostic aside, all of these can be easily implemented and developed into a mobile recommender system. The growing mountain of information that is available all throughout the world that is physically and technologically around us, makes the decision-making ability of the average user almost impossible because of this information overload. Recommender systems are designed to filter through that mountain of data to provide the best suggestions for the current questions being investigated. The increase in micro-computing power aided by cloud computing has taken mobility to the next level of functionality. This allows the recommendation industry to develop a powerful, effective and efficient tool both for the end user and for the companies and institutions themselves. For example, these solutions are becoming three dimensional in that the information applies to multi-dynamics. Another example is a mobile recommender system in the tourism industry, which can be used to provide point-of-interest and context related information to users for trip related decision-making. However, the individual's rights to privacy, security of personal information, and lack of standardization will hinder the widespread adoption and use of the mobile recommender system technology.

Because we are so inundated with information every day, a recommender system can simplify a purchaser's pursuit of products to buy or a location to find one. Often times we all make decisions without having a personal experience and sometimes we rely on the experience of others to make those decisions. With so much information involved in online transactions there is help available through recommender systems. We see this when we use a website or an app via smartphone using Google, or YellowPages.com, and even a travel application like AAA. Recommenders will help users to find a particular set of items based on their preference, because behind the scenes the systems use multi=threaded systems and databases. We see this in today's world through the use of cloud computing and with using Hadoop big data systems. With these trend setting technologies companies boost sales as well as to help individuals to maybe even find new friends or even a relationship. Recommenders require information based on locations, demands, or preferences of a user. What people don't realize is that the information that is gathered using a recommender system can be automatic or it can be via questions asked by the user. You may notice when visiting eBay or amazon pages they may have a list of items at the bottom or to the side of the page that states items you may like. Google also shows personalized advertisements based on previous searches. Also, Google searches are unique to the users' and locations and times. So if you search "red car" today at 1:00 pm you may see a red Ferrari. 
However, at 1:02 pm you may see a red Volvo. The behind the scene search dynamics calculates the "best" recommendations. Recommender systems are important tools for ecommerce companies as it assists management to determine how consumers rate their products. If the product is not among the top rated brands, then why not. Recommender systems also create a privacy concern. Some users are concerned about others knowing their habits. Users may not trust in how their information is shared using recommender systems and may consider it a privacy breach.

The purpose of this research is to analyze and evaluate the use of recommender systems. This research is to identify the potential opportunities and challenges of using recommender systems as applied to the individual's search for the best graduate school program, as well as the effects, both short and long term, of any decisions made using this approach.

\section{LITERATURE REVIEW}

With the ever-increasing amount of information available, the users of that information need some way of overcoming the immense information overload when trying to wade through and make decisions. This is an opportunity for recommender systems to come into play. Recommender systems have been defined in the current literature as "any system that produces individualized recommendations as output or has the effect of guiding the user in a personalized way to interesting or useful objects in a large space of possible options" [2]. Recommender systems gather their knowledge from one or more sources including social (opinions, behavior, etc.), individual (demographics, constraints, etc.), or content (item features, contextual information, etc.) and normally employ two techniques for making recommendations, collaborative and content-based. Collaborative recommendations are simple because the system just matches different users with products that other users have liked or purchased that also have similar interests. Content-based recommendations are slightly more complex because the system must learn the user's preferences before it can make appropriate suggestions based on other user's with similar preferences and the appropriate item's features [2].

Even though the initial objective envisioned for recommender systems was to be similar to a personal assistant and provide the best overall option(s), current recommender systems are biased toward the business goals instead of the user's needs. Just imagine the recommender system at Amazon.com sending you to the local library to check out a book you are looking for instead of purchasing the same book they have in stock. That would be a good example of a recommender system that is biased toward the business and will not provide the best overall choice(s) for the user. Amazon's system is working for the business goal of creating revenue and would never suggest the local library over selling the book. The future of recommender systems should focus on the user's long term goals, take advantage of cloud-based storage and scalability, and provide more contextually pertinent recommendations [6].

Retail e-commerce is the dominant industry that relies on recommender systems to boost their profits through upselling and suggestive selling. However, media websites, advertising firms, and tourism related organizations are beginning to gain interest [1]. Although online businesses are certainly the best vehicle to utilize the current recommender systems, the brick-and-mortar retail stores are trying to join the wave and stay competitive with their online retail counterparts. Through the use of Radio-frequency Identification (RFID) tags on all the products in the store and small computers attached to the shopping carts, shoppers can load in a shopping list which the computer will map out for the shopper. This system also has the ability to receive coupons or offers depending on current store location, provide a running total of items that are placed in the shopping cart, and present possible options that the shopper may have forgotten or did not realize they needed [9].

The pervasiveness of technology in our everyday lives, assisted by the miniaturization and mobilization of computing devices, has propelled the interest and use of recommender systems in other industries including the travel and tourism industries. The tourism industry is a multi-billion dollar industry that could certainly take advantage of the recommendation systems industry and provide better, more accurate trip planning. In addition, mobile services can make the day to day trip experience more enjoyable. Travel planning, available transportation modes, reservation services, Internet search capability, local health and safety information, and context-aware services are the most important categories of services that travelers need. The next level of recommender systems will have both desktop and mobile versions of their interface systems available to supply these services [5]. Smartphones, tablets, laptops, and automobiles are the mobile devices that will carry these services to the masses 
and through standardization and ethical development, these mobile recommender systems will be able to deliver unbiased suggestions without compromising privacy or personal information security.

\section{RESEARCH METHOD}

Research on recommender systems is based on two distinct principles, the level of personalization and whether the system is biased toward the user or business. Personalization is important because over time a user's preferences change, so the recommender system algorithm must take those changes into account when providing suggestions. Bias toward the business will provide greater profits for the business, but may ignore some of the obscure items in the database that the user may be interested in. Bias toward the user would provide the user with the best options available from the business, but may not give the user the best overall option if the system is proprietary. The systems can also be evaluated through the prediction accuracy and the precision of recommendation lists [2].

Past research in the tourism recommendation systems included matching a list of known travel needs with trip planning websites. It also provided comparison among the trip planning websites. This was done in order to determine the best services to offer travelers during their pre-trip planning phase, developing an in-car system to test distractibility and functionality, and statistical modeling of survey questions to determine the most desired services [5]. The research methodology of this paper will be to use current literature and research studies to discuss the current problems facing mobile recommender systems and provide some solutions and future research for consideration.

\section{PROBLEM ANALYSIS}

The first problem to consider with mobile recommender systems is the privacy concerns of the average user. The concern over privacy is normally considered when personal information has been made available to others with or without the user's consent, but there is another perspective. This perspective takes into account the ubiquitous nature of computing into the lives of everyone and how it may cause the loss of existential autonomy or the ability to show a different face in various situations. The ability of cameras to capture our face for facial recognition; biosensors to detect our body chemical compositions; or RFID tag readers to identify products or information from mobile devices; take personal information from us without our consent. This is another perspective of privacy other than address, phone number, or social security number [7]. Privacy is certainly a major concern for tourists as well because they may be travelling to countries that do not have the same protections that the U.S. laws provide them.

The second problem to consider for mobile recommender systems is the security of personal information. The most obvious security threat to personal information is unethical use or acquisition usually by illegal means such as identity theft or hacking. However, there are other more obscure ways to disclose or lose personal information. When a consumer signs up for a website or purchases something online, their personal information is used by those companies, but that information could be sold to other parties, stolen by employees or hackers, or accidentally exposed online. Unsolicited emails are the most recognized form of nuisance that occurs because of those issues [7]. Of course tourists will not need to only worry about U.S. issues and laws regarding private information, they must also deal with the lack of laws in other countries that could potentially use their information at will.

The third and final problem to consider for mobile recommender systems currently is the lack of standardization across all platforms so the distinct recommender systems can communicate and provide the best options for the users. The personalization of the mobile devices such as the Apple iPhone or Android Tablets and the operating system software such as Windows or Linux could have negative effects on the standardization of recommender system input and output mechanisms which could also decrease the adoption and usage of these systems or slow propagation of the required data for users [3]. The automobile platform is probably the most important system that must be standardized at least in communicating between vehicles and land based Internet connections. In addition, the user interface must be kept simple and easy to read or the digital voice must be easy to understand so the driver is not distracted from their main function behind the wheel [4]. Within the tourism industry, the other major hurdle in standardization is trying to get varying governments from different areas of the world to agree on standards, while constrained by cultural nuances and foreign laws. 


\section{Recommended Designs, Solutions and Research}

Through the initial waves of recommendation systems, the cost to purchase the IT equipment and software licenses was extremely prohibitive, but because of the increased usage and interest in the systems, the costs are coming down. This next wave of recommender systems will need to derive more accurate and timely contextual related information from the user's surroundings to provide more precise recommendations when time of day, day of week, weather, and biometrics are taken into consideration. The new systems will also need to focus on the user's longterm goals including budgets and schedules instead of only providing short-term solutions such as suggestive selling or up-selling which is biased toward the business only to improve profit margins. Since the costs of hardware and software are decreasing coupled with the ability to utilize cloud-based vendors to take advantage of unlimited scalability; distributed computing platforms will provide an increased number of companies to offer some form of recommendation service for their customers [6].

For the automobile traveler, the current recommender system designs are mostly targeted at navigation and traffic situations, but since a majority of the adult population spends a great deal of time in the car, the development of fullfeature recommendation systems and communication between vehicles are becoming a reality. One fairly current study developed an in-car system that worked with the navigation system to offer suggestions for local restaurants, hotels, and points-of-interest, but it required a connection to a server through a network connection or a physical data CD with the same database files in the car. The system used a questionnaire to populate the initial user preferences and used an algorithm to update those preferences as the user made choices so the system could change along with the user's preference changes, but the $\mathrm{CD}$ was static until the developers created another CD with the updates. The application could also have multiple users set up for a single vehicle because they used smart cards with a chip that had the user's system ID number encoded on it and the user plugs it into the system to begin [3].

The ability for vehicles to communicate with each other or provide each other with network connectivity for Internet related content is slow to gain interest mostly because the high number of different perspectives within the automotive manufacturing industry keeps standardization low on the priority list. Standards need to be developed to ensure that no matter which vehicle you own or are driving, the system will know the user's preferences, be able to gather the contextual information accurately through a standard network connection, and be able to display visually or audibly the information in a way that does not distract the driver. Even if the systems themselves are proprietary, the automobile manufacturers need to ensure that the communication, processing, and contextual display methods (visual or audible) are standardized across the entire industry [4].

The life cycle of an average tourist has a pre-trip phase, an on-trip phase, and a post-trip phase. The pre-trip phase is usually where a majority of travelers will determine their itinerary, accommodations, and transportation needs and there are currently plenty of websites (Orbitz.com, Kayak.com, etc.) that can assist the traveler with all of these steps. Other than recommending hotels or rental cars for the specific trip location, they are limited to basic recommendations and mostly desktop computer applications instead of complex analytical choices based on contextual parameters and user preferences along with a lack of mobile applications. There are not many applications for on-trip services, but the interest is growing and the most prominent services that are requested are: selection of points-of-interest based on preferences; route planning and transportation mode available; better trip cost estimation that includes costs for points-of-interest admission; and the ability to change plans during the trip. The post-trip phase is the most social of the phases because the majority of people enjoy sharing their photos or experiences online through Facebook, Twitter, or travel websites. The post-trip data can then be used in recommender systems to give the pre-trip and on-trip travelers better information to make more precise decisions [8].

\section{Analysis and Evaluation of Recommender Systems as applied to the Search for Graduate Programs}

This section evaluates recommender systems and their facility of searching for a graduate program that is best suited to an individual's interests, abilities, and limitations. One theme that is recurring in all of the research I've done surrounding recommender systems is that much of their value lies within their ability to help users mitigate the ever increasing problem of being overwhelmed with the amount of data and choices available on the internet today. The search for a graduate program is no exception to this problem. Most people have certain attributes in mind when 
they begin their search for a graduate program, such as whether they want a full time, part-time classroom, or parttime online environment, the general field of study, and sometimes even the location. Beyond these basic identifiers, most people need assistance in filtering out all of the choices and understanding the differences between these choices yet there was no system that went beyond these high level factors. For example, www.gradschools.com allows you to choose subject, specialty, format, and location as a type of filtering aid in your search for potential schools, even going so far as to list majors on the left side as a secondary filter once the first search is completed, but beyond that the decision is left entirely up to the user and how many of these options they may want to further investigate. This is an example of a fairly basic form of content-based filtering. It is using your answers to four categories previously mentioned as the discrete characteristics it will reference to compare to those same four characteristics and the value for each that has been assigned to all of the possible graduate programs in their database. It will then select the programs that match those criteria for your further evaluation. While this may provide some guidance and direction where previously the individuals search had none, it fails to factor in many other attributes or information that may be able to enhance the relevance of the results to the user and increase their satisfaction and trust in those results presented to them.

The Technology Acceptance Model is a business intelligence model that evaluates factors such as the perceived usefulness and perceived ease of use in determining how a user will react to and accepts new technology. This is key to designing any effective recommender system because despite how quick, accurate, or pertinent the results may be, if the user does not find the system easy enough to use and the results are not found and displayed in such a way that the user has trust in these results than ultimately the system has failed to achieve its purpose. Keeping this in mind I think the best approach to finding the graduate program most suited to each individuals needs and interests is one that uses a hybridized approach between content-based and collaborative filtering while utilizing an example critiquing interface with fish eye view. The content-based filtering portion of the solution will involve a similar initial questioning as gradschools.com, but would expand upon that, asking more in depth questions including things like preferred price ranges, and opening up secondary questioning dependent on your answer to the initial attributes. For example, whether or not you choose an on campus, online, or combined program will yield a different set of follow up questions that are more specific to those options. The collaborative filtering would be a secondary aspect that's usefulness increases over time and with the number of users that have already completed the search. Users would answer individual surveys with questions regarding their backgrounds, interests, previous study, etc and that data as well as the results of those individuals' searches and selections will be stored for comparison with future users. So a secondary consideration to the initial content based questions will be the recommender system evaluating whether there are previous users with similar characteristics to you that have already used this search and incorporating potential results and information learned through them. This way the system has the potential to learn and incorporate user experiences in future searches to enhance the results for future users.

The results of this recommender system can have effects on both the individuals and the schools. While a tool like this can help students to explore options they may not have found themselves through simple search methods, if not structured and properly tested it could eliminate certain choices that may have provided the best solution for the student. Many people assume recommender systems will be accurate and effective, and considered all the possible solutions so if this is not actually happening and people make that assumption they are being limited in their possible outcomes. It also empowers the user to investigate the growing number of options and not be forced to accept the costs or limited selections of certain schools because they are the only options they were aware of or could find information on. This in turn forces schools to continually have to strive to be competitive and well rated programs in order to obtain the "business" or enrollment of these students.

The biggest challenge in designing a system to achieve this would be collecting all of the initial data and assigning the attributes to the schools in order to perform the first content-based filtering described above. Much effort would have to be put into finding every possible graduate school program available and documenting the specific attributes for each of these universities that the system will evaluate and compare against one another. If this is not done thoroughly, again there is the potential to limit options presented to a student and the best fit for them may not be included in the results. 


\section{CONCLUSIONS}

Recommender systems are clearly a necessary and important tool in dealing with the "information overload" being experienced by individuals in today's world whether in personal or business related situations. The potential applications of and value in recommender systems vary across industries and is highly dependent on the availability of data in one form or another, which with today's technology and hardware solutions is rarely an issue. The focus and goal of these systems as well as their complexity has increased over time and is reflected in the research found within the articles that were reviewed. I think recommender systems increased application to the search for a graduate school best suited to each individual would be a highly valued use of the tool and something that is somewhat lacking predicated on research.

Consciousness is important for security complications with recommender systems, being open on how the system operates as it deals with users. Particular sites should clearly state their policies with recommender system as well as the role of those that leave ratings for a given product who then in turn can act as a rating system to your next search. When the risk and the benefits of the recommender system is provided this will increase acceptance for recommender systems; we easily see this as we use Google. Even though you may not have a Google account Google still knows who you are and what you are looking for based on your IP address. This "assistance" at best helps users make informed choices about their level of involvement. Offering incentives for participating will also encourage consumers to be more involved and active leading to greater sharing of information. It would be beneficial to management if they use a collaborative filtering recommender system, this type of recommender system methods are based on collection and analyzing of a large amount of information on users such as behaviors, activities or preferences and predicting what users will like based on their similarity to other users. Collaborative filtering collects the data from willing participants and it's not systematic. This will help to reduce trust issues and privacy concerns as users choose what they want to share.

Although the interest in mobile recommender systems is extremely high, the ability of multiple manufacturers and software developers to standardize the recommender system architecture will only come into reality through some form of coalition between the various companies. The coalition could present guidelines on how the different recommender systems process and share information within their proprietary systems, similar to the methods employed by accountants within the AICPA (American Institute of Certified Public Accountants). The governments of the world must also agree to provide laws and regulations to ensure that each individual user gets the protections and recourse necessary to ensure their privacy and personal information security. The future certainly looks bright for the mobile recommendation market, but unless companies and governments embrace the need to work together, the mobile recommendation market will remain modestly simple.

\section{REFERENCES}

1. Aldrich, S. E. (2011). Recommender systems in commercial use. AI Magazine, 32(3), 28-34. Retrieved from http://search.proquest.com/docview/908412539?accountid=35796

2. Burke, R., Felfernig, A., \& Göker, M.,H. (2011). Recommender systems: An overview. AI Magazine, 32(3), 13-18. Retrieved from http://search.proquest.com/docview/908412537?accountid=35796

3. Console, L., Torre, I., Lombardi, I., Gioria, S., \& Surano, V. (2003). Personalized and adaptive services on board a car: An application for tourist information. Journal of Intelligent Information Systems, 21(3), 249.

Retrieved from http://search.proquest.com/docview/200209968?accountid=35796

4. Gerla, M. \& Kleinrock, L. (2011). Vehicular networks and the future of the mobile internet. Computer Networks. doi:10.1016/j.comnet.2010.10.015

5. Goh, D. H., Ang, R. P., Lee, C. S., \& Lee, C. K. (2010). Determining services for the mobile tourist. The Journal of Computer Information Systems, 51(1), 31-40. Retrieved from

http://search.proquest.com/docview/808409733?accountid=35796

6. Martin, F. J., Donaldson, J., Ashenfelter, A., Torrens, M., \& Hangartner, R. (2011). The big promise of recommender systems. AI Magazine, 32(3), 19-27. Retrieved from $\mathrm{http} / / /$ search.proquest.com/docview/908412542?accountid=35796

7. Michelfelder, D. P. (2010). Philosophy, privacy, and pervasive computing. AI \& Society, 25(1), 61-70. doi:http://dx.doi.org/10.1007/s00146-009-0233-2 


\section{Issues in Information Systems}

Volume 15, Issue II, pp. 168-174, 2014

8. Sylejmani, K., \& Dika, A. (2011). A survey on tourist trip planning systems. International Journal of Arts \& Sciences, 4(9), 13-26. Retrieved from http://search.proquest.com/docview/929269885?accountid=35796

9. Walter, F. E., Battiston, S., Yildirim, M., \& Schweitzer, F. (2012). Moving recommender systems from online commerce to retail stores. Information Systems and eBusiness Management, 10(3), 367-393.

doi:http://dx.doi.org/10.1007/s10257-011-0170-8 